MODELING, IDENTIFICATION AND CONTROL, 1993, VOL. 14, NO. 4, 193-218

doi:10.4173/mic.1993.4.2

\title{
ALSPEN-A mathematical model for thermal stresses in direct chill casting of aluminium billets
}

\author{
HALLVARD G. FJÆR† and ASBJØRN MO $\ddagger$
}

Keywords: DC casting, aluminium, stress modeling.

\begin{abstract}
This paper presents the mathematical model ALSPEN, in which the thermally induced strains and stresses which develop during direct chill (DC) semicontinuous casting of aluminium billets are calculated by a finite-element method. The metal is assumed to be an isotropic elastic-viscoplastic material with strongly temperaturedependent properties. In the material description, the viscoplastic strain is treated in a 'unified' manner, in which low-temperature (approximately) time-independent plasticity and creep at high temperatures occur as special cases. Furthermore, in the numerical time stepping procedure, all of these plastic material properties which are present simultaneously in the solution domain as a result of the large temperature differences, are treated in a similar way. To demonstrate some of the capabilities of ALSPEN, we have modeled the casting of an AlMgSi alloy, AA6063. The material properties of this alloy have been studied in parallel with the development of the mathematical model.
\end{abstract}

\section{Introduction}

Direct chill (DC) semicontinuous casting of axisymmetric billets is one of the most important processes in the production of aluminium. A major problem, however, is the formation of residual stresses which can cause cold cracking. Furthermore, thermally induced stresses and strains can lead to defects during casting, and especially close to the liquid zone, this may result in hot tearing.

The purpose of this paper is to present the mathematical and numerical model ALSPEN. In this model, the stresses and strains which develop during casting are calculated in order to predict the optimal process parameters and thereby avoid defects in the ingot.

In parallel with the development of ALSPEN, the viscoplastic material properties of the AlMgSi alloy AA6063 have been studied in Nedreberg (1990)§ and these properties are incorporated in the present version of ALSPEN.

A successful solution of the residual stress problem depends upon a correct solution of the thermal problem. For the present study, the thermal problem has been solved by the temperature model ALSIM-2 in which the heat transfer during casting is simulated. This model is described in Madsen and Fladmark (1973) and verified in Madsen (1979), Fossheim and Madsen (1979), Vorren and Brusethaug (1987), Jensen (1980), Jensen and Schneider (1990).

Several models for simulating thermal stresses in continuous casting have been reported, and two-dimensional (2-D) models where either plane stress or plane strain is

Received 11 May 1993.

† Institutt for energiteknikk, 2007 Kjeller, Norway.

†SINTEF SI, 0314 Oslo 3, Norway.

$\S$ This PhD. thesis, which was written in English at the University of Oslo, will be made available to the public during 1990/91.

Reprinted, with permission, from Metallurgical Transactions B, Volume 21B, December 1990. 
assumed are presented in Kristiansson (1982), Williams, Lewis and Morgen (1979), Rammerstorfer, Jaquemar, Fisher and Wiesinger (1979).

The case of axisymmetric stress modeling of DC cast aluminium is studied in Moriceau (1975), Mathew and Brody $(1976,1979)$. The important rate dependence of the constitutive equations, however, is not taken into account in Mathew (1979). In Mathew $(1976,1979)$, on the other hand, constitutive equations for both timeindependent plastic strain and creep are implemented, but the papers do not give a detailed study of the material behaviour.

Other relevant papers to the present problems are Smelser and Richmond (1988), Thomas, Samarasekera and Brimacombe (1987). The solidification of a cylindrical casting is studied in Smelser and Richmond (1988). Here, the thermal and stress problems are solved, and the viscoplastic material model with one internal variable, given in Sample and Lalli (1987), is used in the stress problem. Thomas et al. 1987), describes a mathematical model which calculates the thermal stresses generated in an ingot during solidification, and a rather general form of the constitutive equations, including a 'structure parameter', is discussed. In the calculations, however, the viscoplastic part of the strain is described only by a creep formula.

In the present study, we have treated the problem in a transient manner, leading to a continually increasing solution domain consisting of the solid part of the billet. In the finite-element solution, we must, at certain time steps, incorporate new elements in the solution domain, and it turns out that an initial component of the strain field must be added to each new element in order to ensure that the displacement and stress fields shall not be disturbed by its incorporation. It should be noted that this initial strain component is significant for the quantitative results.

In our material description, the viscoplastic strain has been treated in a 'unified' manner as one quantity, and our theory does not require a yield criterion or loading and unloading conditions. For the AlMgSi alloy AA6063, we have found explicit mathematical formulae for the relation between effective flow stress, temperature, and effective viscoplastic strain rate. In this relation, we have also included a hardening parameter by which the strain hardening in the metal is quantified.

Section 2 of the present paper concerns the formulation of the mathematical model, and in Section 3, we present the numerical solution. Results obtained by ALSPEN are discussed in Section 4.

\section{Model formulation}

Because of the axisymmetric form of the billet, the mathematical problem reduces to two dimensions, and we denote the radial and axial coordinates by $r$ and $z$, respectively. The region, $\Omega$, in which the problem is to be solved is shown in Fig. 1, together with the frame of reference.

The stress distribution in the solid part of the billet is governed by Cauchy's equations, which will be solved by a finite-element technique. As is common in finiteelement analysis, we therefore start from the statement of virtual work

$$
\int_{\Omega} \delta \varepsilon^{*} \cdot \sigma d \Omega-\int_{\Omega} \delta \mathbf{u}^{*} \cdot \rho \mathrm{g} d \Omega-\int_{\mathbf{r}} \delta \mathbf{u}^{*} \cdot \mathbf{t} d \Gamma=0
$$

Here, the superscript '*' denotes the transpose of a vector (or matrix), $\mathbf{g}$ is the acceleration of gravity, $\rho$ is the density, and $t$ is the force per unit length acting on the boundary $\Gamma$. Furthermore, $\delta \mathbf{u}$ and $\delta \varepsilon$ denote virtual variations of displacement $\mathbf{u}$ and associated strain vector $\varepsilon$, respectively. Expressed by its radial and axial components $u_{r}$ 


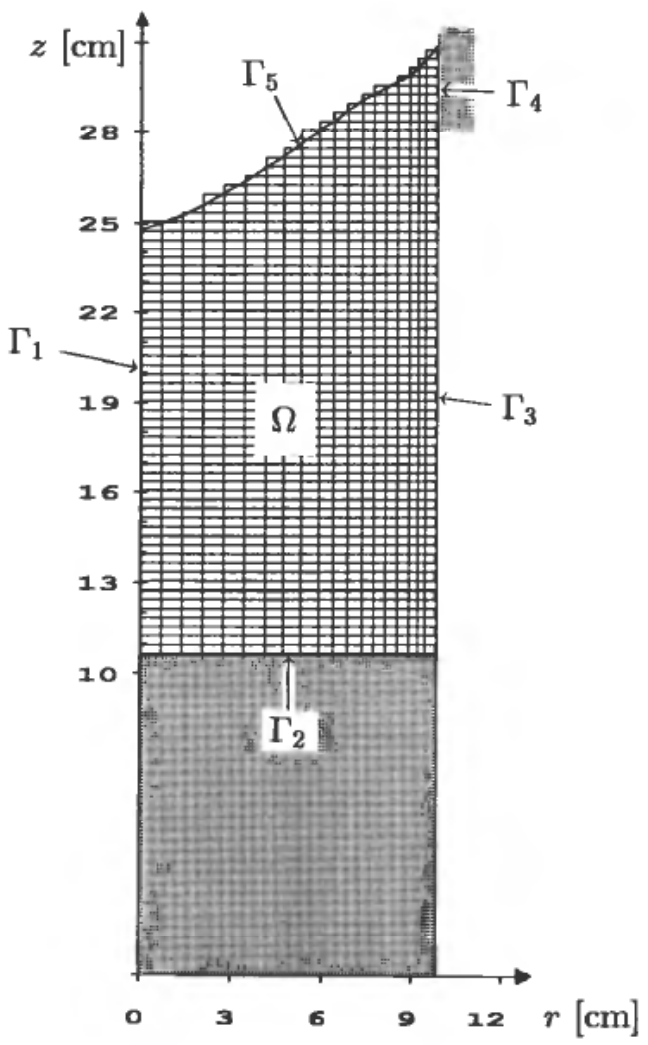

Figure 1. Solution domain and finite-element mesh.

and $u_{z}$, the displacement field is given by

$$
\mathbf{u}(r, z)=\left[\begin{array}{l}
u_{r}(r, z) \\
u_{z}(r, z)
\end{array}\right]
$$

and the relation between $u$ and $\varepsilon$ can be written as

$$
\boldsymbol{\varepsilon}=\mathbf{B} \cdot \mathbf{u}
$$

where

$$
\mathbf{B}=\left(\begin{array}{cc}
\frac{\partial}{\partial r} & 0 \\
\frac{1}{r} & 0 \\
0 & \frac{\partial}{\partial z} \\
\frac{1}{2} \frac{\partial}{\partial z} & \frac{1}{2} \frac{\partial}{\partial r} \\
\frac{1}{2} \frac{\partial}{\partial z} & \frac{1}{2} \frac{\partial}{\partial r}
\end{array}\right)
$$


Finally, the associated stress vector, $\sigma$, and the associated strain vector, $\varepsilon$, are given by

$$
\sigma=\left(\begin{array}{c}
\sigma_{r} \\
\sigma_{\theta} \\
\sigma_{z} \\
\tau_{r z} \\
\tau_{z r}
\end{array}\right)
$$

and

$$
\varepsilon=\left(\begin{array}{c}
\varepsilon_{r} \\
\varepsilon_{\theta} \\
\varepsilon_{z} \\
\varepsilon_{r z} \\
\varepsilon_{z r}
\end{array}\right)
$$

The subscripts $r$, and $\theta$, and $\mathrm{z}$ denote the radial, circumferential, and axial directions, respectively, and the shear strain and shear stress have subscripts $r z$ and $z r$.

\section{2(A) Boundary conditions}

As indicated in Fig. 1, the boundary $\Gamma$ is subdivided according to the different physical conditions applying. Due to the axial symmetry, the radial displacement and the shear stress equal zero on the centreline, $\Gamma_{1}$.

At the bottom, $\Gamma_{2}$, we have assumed that the billet can move freely in the positive axial direction and without surface friction in the horizontal direction. The boundary $\Gamma_{4}$ is treated in a similar manner. Here, the billet can move freely in the negative radial direction, and there is no friction in the axial direction. Furthermore, a free surface condition is implemented at $\Gamma_{3}$.

The boundary $\Gamma_{5}$ is defined by the isotherm for temperature, $T_{c}$. The term $T_{c}$ is the so-called coherency temperature, being defined as the temperature above which the alloy is treated like a liquid and below which it is treated like a solid. Since coherence depends on the skeleton resulting from physical bridging of the dendrites, it is difficult to specify an exact numerical value of $T_{c}$ for a given alloy. It lies between the liquidus temperature and the temperature of final solidification, which equals the temperature of a (meta)stable eutectic. For the AlMgSi alloy AA6063 studied in the present paper, $T_{c}$ is given the value $913 \mathrm{~K}\left(=640^{\circ} \mathrm{C}\right)$. Above $\Gamma_{5}$, the liquid melt acts with hydrostatic pressure, and this boundary will be more thoroughly discussed in Section 3(A).

\section{2(B) Constitutive equations}

The metal is described as an isotropic elastic-viscoplastic material in which strain is generated by thermal contractions. While the material parameters are strongly dependent on temperature, the temperature increase generated by the viscoplastic strain is negligible. A coupled thermoviscoplastic description of the material is therefore not necessary. Furthermore, we have based our mathematical formulation on the classical small deformation theory (this assumption is justified by the results obtained by ALSPEN) implying that the total strain may be divided into an elastic, a 
viscoplastic, and a thermal part, which will be denoted here by superscripts $e, p$, and $T$, respectively. Hence,

$$
\varepsilon=\varepsilon^{e}+\varepsilon^{p}+\varepsilon^{T}+\varepsilon^{i}
$$

where we also have introduced the so-called initial component, $\varepsilon^{i}$, of the associated strain vector mentioned in the Introduction. This part of the strain will be discussed in Section 3 (B).

The thermal contractions can be expressed by an associated thermal strain vector given by

$$
\varepsilon^{T}=-\left(\begin{array}{l}
1 \\
1 \\
1 \\
0 \\
0
\end{array}\right) \int_{T}^{T_{c}} \beta(T) d T
$$

where $\beta$ is the coefficient of thermal expansion. The temperature dependence of $\beta$ has been taken from Mondolfo (1976).

The elastic part of the strain is given by Hooke's generalized law, which here is written in the form

$$
\boldsymbol{\sigma}=\mathbf{D} \cdot \boldsymbol{\varepsilon}^{e}
$$

where

$$
\cdot\left(\begin{array}{ccccc}
1-v & v & v & 0 & 0 \\
v & 1-v & v & 0 & 0 \\
v & v & 1-v & 0 & 0 \\
0 & 0 & 0 & 1-2 v & 0 \\
0 & 0 & 0 & 0 & 1-2 v
\end{array}\right)
$$

The temperature dependence of Young's modulus, $E$, and Poisson's ratio, $v$, has also been taken from Mondolfo (1976).

\section{Viscoplastic strain}

In material descriptions for problems related to ours, creep and time-independent plastic flow are very often treated separately by so-called overlay models (Mathew and Brody 1976). For a basic discussion of overlay models, we refer to Chapter 8.15 in Owen and Hinton (1980). From a mechanical point of view, however, the strain generated by creep is indistinguishable from that resulting from time-independent plastic flow, and particularly at higher temperatures, only the combined effect is experimentally observable (Thomas et al. 1987, Zienkiewitcz and Cormeau (1974)).

In ALSPEN, we have therefore treated the 'total' viscoplastic strain as one quantity, as is usual in 'modern' constitutive equations with internal variables. Furthermore, we have in the present version of the model approximated the material behaviour by introducing a hardening parameter which accounts for the isotropic strain hardening 
of the material. In this description, kinematic hardening is not taken into account, partly due to lack of experimental data for the actual alloy (AA6063). (As will be discussed below, only tensile tests have been carried out in Nedreberg (1990).) It should also be noted that a large part of the viscoplastic deformation occurs in the "hightemperature' regime, in which hardening is of minor importance.

Recent applications of internal variable constitutive equations are presented in Smelser and Richmond (1988), Brown, Kim and Anand (1989), Makin, MacEwen, Winter, Mason, Kanouff and Fuchs (1988). In Smelser and Richmond (1988), where the development of thermal stresses in a solidifying cylindrical casting is modeled, the constitutive equations are valid for commercially pure aluminium. Hot compression tests of an iron- $2 \%$ silicon alloy and commercially pure aluminium are simulated in Brown et al. (1989) and Makin et al. (1988) internal variable constitutive equations for 304 stainless steel, developed by Bammann (1988), are applied in the evaluation of residual stresses in a travelling gas tungsten arc (GTA) weld. However, any papers using an internal variable constitutive theory in modeling the solidification of an AlMgSi alloy are not known to us.

We have assumed the viscoplastic (nonelastic) deformation to be incompressible, and the viscoplastic strain increment, $d \varepsilon^{p}$, to be given by Prandtl-Reuss relations for associated plasticity

$$
d \varepsilon^{p}=\frac{3 d \bar{\varepsilon}^{p}}{2 \bar{\sigma}} \cdot \sigma^{\prime}
$$

Here, $\sigma^{\prime}$ denotes the associated deviatoric stress vector, and the effective stress, $\bar{\sigma}$, and the effective viscoplastic strain increment, $d \bar{\varepsilon}^{p}$, are defined by

$$
\bar{\sigma}=\left(\frac{3}{2} \sigma^{\prime} \cdot \sigma^{\prime}\right)^{1 / 2}
$$

and

$$
d \bar{\varepsilon}^{p}=\left(\frac{2}{3} d \varepsilon^{p} \cdot d \varepsilon^{p}\right)^{1 / 2}
$$

respectively.

In 'classical' (time-independent) plasticity theory, isotropic strain hardening is taken into account by an empirical relation between the effective stress, $\bar{\sigma}$, and the integrated effective plastic strain increment, $\int d \bar{\varepsilon}^{p}$. However, in order to incorporate the dependence on temperature and strain rate in the theory, we have, in accordance with Persyna (1966), also introduced the effective viscoplastic strain rate, $\bar{\varepsilon}^{p}=d \bar{\varepsilon}^{p} / d t$, and the temperature, $T$, in this relation. Furthermore, we have only taken the strain hardening into account below some certain temperature $T_{0}$, since all the viscoplastic strain may be assumed to be generated by creep above $T_{0}$ (Nedreberg 1990). Consequently, the above-mentioned hardening parameter, $\alpha=\int d \alpha$, is defined by

$$
d \alpha= \begin{cases}d \bar{\varepsilon}^{p} & \text { when } T \leqslant T_{0} \\ 0 & \text { otherwise }\end{cases}
$$

Viscoplastic material properties are specified for the AlMgSi alloy AA6063 in Nedreberg (1990). For representative constant values of $T$ and $\bar{\varepsilon}^{p}, \bar{\sigma}$ here has been related to the accumulated viscoplastic strain (which is equal to $\alpha$ when $T$ is below $T_{0}$ ) in a series of tensile tests. The main problem in experiments of this kind is to obtain a 
microstructure in the test specimen approximately equivalent to that during casting. In Nedreberg (1990), this problem is treated as follows. In experiments at temperatures higher than $300^{\circ} \mathrm{C}$, the temperature is first elevated to approximately $550^{\circ} \mathrm{C}$ and then lowered as fast as possible to the experimental value. On the other hand, in the "lowtemperature experiments' $\left(T\right.$ below $\left.300^{\circ} \mathrm{C}\right)$, the temperature is increased as fast as possible to the experimental temperature.

Assuming $\bar{\sigma}$ to be dependent on $\alpha, \bar{\varepsilon}^{p}$, and $T$ only, it has been found appropriate to fit the experimental curves by

$$
\bar{\sigma}=f\left(\alpha, \bar{\varepsilon}^{p}, T\right)=c(T)\left(\alpha+\alpha_{0}\right)^{n(T)}\left(\bar{\varepsilon}^{p}\right)^{m(T)}
$$

where $c, n$, and $m$ are nonlinear functions of $T$. The values of $c(T)$ and $n(T)$ decrease when $T$ increases, and they are fitted by

$$
c(T)=a_{c}\left(1-b_{c} \cdot \tanh \left(\frac{T-d_{c}}{c_{c}}\right)\right)
$$

and

$$
n(T)=a_{n}\left(1-b_{n} \cdot \tanh \left(\frac{T-d_{n}}{c_{n}}\right)\right)
$$

respectively. The value of $m(T)$ increases with $T$ and fitted by the 4th degree polynomial

$$
m(T)=P_{0}+P_{1} T+P_{2} T^{2}+P_{3} T^{3}+P_{4} T^{4}
$$

$\alpha_{0}$ is a constant equal to 0.001 , and the 'onset temperature' $T_{0}$, above which the strain hardening can be neglected, has been determined to be $700 \mathrm{~K}$. The 'material constants' in the three equations above are easily determined by least-squares fit to the results given in Nedreberg (1990), and their numerical values are given in Table 1.

It is well known that the mathematical formula (Eqn. (15)) also applies to aluminium alloys other than AlMgSi AA6063. For some alloys, however, it may be appropriate to use other functional forms for $c(T), n(T)$, and $m(T)$ than those applied in the present case, and in Section 3(C), it will be shown that, in principle, any form of $f$ which is differentiable with respect to $\alpha$ and $\bar{\varepsilon}^{p}$ may be applied in ALSPEN. It should furthermore be noted that an elaboration of the material description by introducing more general constitutive equations with several internal variables (see, for example, Miller (1987) and references cited there) is, mathematically and numerically, relatively simple in ALSPEN.

In the mathematical description of the viscoplastic strain, the commonly used concept of 'static yield stress' Perzyna (1966) has not been introduced. In the material description, we have only defined a flow strees, $\bar{\sigma}$, which depends on the effective viscoplastic strain rate (and the temperature). This means theoretically that a viscoplastic strain will be generated for all nonzero values of $\bar{\sigma}$ at any temperature. At low temperatures, however, the viscoplastic strain rate will be very close to zero for low

Table 1. Viscoplastic material constants.

\begin{tabular}{lll}
\hline$a_{c}=160[\mathrm{MPa}]$ & $a_{n}=0.102$ & $P_{0}=4.41 \times 10^{-2}$ \\
$b_{c}=0-89$ & $b_{n}=1.04$ & $P_{1}=-4.16 \times 10^{-4}\left[\mathrm{~K}^{-1}\right]$ \\
$c_{c}=108[\mathrm{~K}]$ & $c_{n}=135[\mathrm{~K}]$ & $P_{2}=1.45 \times 10^{-6}\left[\mathrm{~K}^{-2}\right]$ \\
$d_{c}=487[\mathrm{~K}]$ & $d_{n}=533[\mathrm{~K}]$ & $P_{3}=-1.84 \times 10^{-9}\left[\mathrm{~K}^{-3}\right]$ \\
& & $P_{4}=1.08 \times 10^{-12}\left[\mathrm{~K}^{-4}\right]$ \\
\hline
\end{tabular}


values of $\bar{\sigma}$, resulting in negligible viscoplastic strain. For a more thorough discussion, we refer here to a recent paper by Lush et al. (1989).

It should finally be noted that in the mathematical limit of time independency (i.e., $m=0$ in Eqn. (15)), a yield criterion has to be incorporated into the constitutive equations. This special case is further discussed in Section 3(C).

\section{Numerical solution}

\section{3(A) Finite-element formulation}

After some elementary algebra, the following expression may be derived from the equations in Section 2:

$$
\begin{aligned}
\int_{\Omega}(\mathbf{B} \cdot \delta \mathbf{u})^{*} \cdot \mathbf{D} \cdot \mathbf{B} \cdot \mathbf{u} d \Omega= & \int_{\Omega}(\delta \mathbf{u})^{*} \cdot \rho \mathbf{g} d \Omega \\
& +\int_{\Gamma}(\delta \mathbf{u})^{*} \cdot \mathbf{t} d \Gamma \\
& +\int_{\Omega}(\mathbf{B} \cdot \delta \mathbf{u})^{*} \cdot \mathbf{D} \cdot \boldsymbol{\varepsilon}^{T} d \Omega \\
& +\int_{\Omega}(\mathbf{B} \cdot \delta \mathbf{u})^{*} \cdot \mathbf{D} \cdot \boldsymbol{\varepsilon}^{i} d \Omega \\
& +\int_{\Omega}(\mathbf{B} \cdot \delta \mathbf{u})^{*} \cdot \mathbf{D} \cdot \boldsymbol{\varepsilon}^{p} d \Omega
\end{aligned}
$$

Here, only the displacement field, $u$, and the viscoplastic part of the associated strain vector, $\varepsilon^{p}$, are unknown.

The finite element formulation of Eqn. (19) leads to a set of equations which formally may be written as

$$
K_{i j} U_{j}=F_{i}^{(g)}+F_{i}^{(t)}+F_{i}^{(T)}+F_{i}^{(i)}+F_{i}^{\left(\varepsilon^{p}\right)}
$$

where $K_{i j}$ are the components of the global stiffness matrix containing information about the elastic properties of the material and $U_{j}$ are the components of the displacement vector in all of the nodes. The right-hand side of Eqn. (20) is divided into five terms corresponding to the terms on the right-hand side of Eqn. (19). The first two terms are given by the acceleration of gravity and the boundary conditions, and the three succeeding terms account for the thermal, initial, and viscoplastic strains, respectively.

\section{3(B) The finite-element mesh}

In the finite-element discretization, we have applied rectangular bilinear isoparametric elements with four nodes, and in the numerical integration of the shape functions, we have applied only one integration point as in Liu, Ong and Uras (1985), Liu, Belytschko, Ong and Law (1985). The efficiency compared to accuracy of different 2-D elements is discussed in Crook and Hinton (1987), where it is shown that the fournode isoparametric bilinear element 'stabilized' with one-point integration (i.e., the 'E41S' element being applied in our present paper) is the most efficient.

An objection to using the rectangular shape is that it is impossible to obtain a close fit between $\Gamma_{5}$, which is the only skew boundary of the solution domain, and the finiteelement mesh. We believe, however, that the error introduced in ALSPEN by this 
inaccurate fit is of the same order of magnitude as the error due to the uncertainty in specifying the coherence temperature $T_{c}$ (Section 2(A)).

Prior to an ALSPEN modeling of stresses and strains, the complete temperature history at any position in the billet is calculated by the model ALSIM-2, which stores the temperature field in prescribed time steps. Between these steps, the temperature field is approximated by linear interpolation.

The stress and strain calculation starts at time $t=t_{0}$, which is the first time step for which the temperature field is calculated. The solution domain is then defined by all elements where one of the following two conditions is fulfilled: (1) at least three nodes are below the isotherm for the coherency temperature $T_{c}$, or (2) two nodes are below this isotherm and the mean temperature of all four nodes is below $T_{c}$. As time proceeds, new elements are incorporated at each time step where one of the conditions given above is fulfilled.

All new elements are given initial values for the viscoplastic, elastic, and thermal strains in the midpoint due to the one-point integration formula of the shape functions. The initial value of the viscoplastic strain is equal to zero, and the initial value of the elastic strain, $\varepsilon_{0}^{e}$, is generated by the hydrostatic pressure acting upon the billet by the liquid zone of the metal. Since the temperature in the midpoint of new elements is generally less than the coherency temperature, we also have an initial value of the thermal strain, $\varepsilon_{0}^{T}$, which is given by Eqn. (8).

When a new element is incorporated in the mesh, the displacement in the new nodes is preset to zero, and nodes shared with 'solid members' of the mesh are known from the calculation for the previous time step. Since the initial value of the total strain, $\varepsilon_{0}$, is related to the displacement $\mathbf{u}$ (Eqn. (3), it is, in general, different from the sum of the (nonzero) initial values of elastic and thermal strains defined above. This problem, however, is easily solved by the introduction of a so-called initial strain component, as mentioned in Sections 1 and 2. This strain component is given by

$$
\varepsilon^{i}=\varepsilon_{0}-\varepsilon_{0}^{e}-\varepsilon_{0}^{T}
$$

and its numerical value is usually much larger than the values of $\varepsilon_{0}^{e}$ and $\varepsilon_{0}^{T}$. The initial strain remains constant during the proceeding time steps of (Eqn. (7)), and we notice that $\varepsilon^{i}$ in some elements has the same order of magnitude as the total strain at the end of casting.

Our approach to the problem of an increasing solid region has much in common with a method used in Kristiansson and Zetterlund (1982), where the liquid zone is also part of the solution domain. The 'liquid elements' here are given zero stiffness, and nodes surrounding exclusively by such elements have displacements prescribed to zero. Another method in which all elements in the liquid zone are given a 'small' stiffness is used in Smelser and Richmond (1988), and Richmond gives a general discussion of the problem in Richmond (1981).

In some cases, it turns out that some regions (in the upper part) of an already solidified billet may remelt. We have modeled this case by resetting the strains and the stresses in the actual element(s) to zero when both conditions (1) and (2) mentioned above are no longer fulfilled and thereafter regarded these regions as part of the liquid melt.

\section{3(C) Time stepping procedure}

As mentioned in Section 2(B), the total viscoplastic strain is traditionally divided artificially into a time-independent plastic part and time-dependent creep. The 
numerical integration of these two components is based on different formulations of the constitutive equations, and normally an initial strain method is used to integrate timedependent equations. This method, however, can give numerical problems due to the inherent stiff characteristics when the constitutive equations are integrated in the low strain sensitivity regime.

In our mathematical model, the constitutive equations are mathematically very similar to those in Tanaka and Miller (1988), where a function similar to our $f$ is introduced. In Tanaka and Miller (1988), this function depends on the effective viscoplastic strain rate and a 'structure variable' (playing the same role as our $\alpha$ ) which determines the strain hardening, and a forward gradient operator is applied to integrate the constitutive equations. Similar noniterative integration methods have been described in Agyris, Vaz and William (1987, Pierce, Shih and Needleman (1984).

Snyder and Bathe (1981) have developed a 'unified' initial strain algorithm in which time-independent plastic strain and creep are treated in a similar manner. Their constitutive equations, however, are based on overlay models of the generated viscoplastic strain. In the present paper, we have developed a related, but more simple, numerical integration algorithm in which the requirements for a unified formulation of the constitutive equations are met. This algorithm, which will be discussed in detail below, has obvious advantages in the DC casting problem, since both time-dependent and time-independent material properties are present simultaneously in the solution domain as a result of the large temperature differences.

Our scheme belongs to a class of iterative predictor-corrector algorithms in which the global system of equations in solved several times on each time step (see, for example, Tanaka and Miller (1988) and Agyris et al. (1978).). The iterations are performed on the increments of the viscoplastic strain, which appears on the right-hand side of the equation system, and the local solution of the constitutive equations is carried out in an approximative, but noniterative, manner at each iteration step.

We denote all quantities at time $t=t_{n}$ and at time $t=t_{n+1}$ by subscripts $n$ and $n+1$, respectively. Assuming all quantities at $t_{n}$ to be known, we must at $t_{n+1}=t_{n}+\Delta t_{n+1}$ solve Eqn. (20) in order to obtain the unknown displacement field $U_{j_{n+1}}$. The last term on the right-hand side of this equation, however, is unknown since it depends on the unknown viscoplastic strain tensor field, $\varepsilon_{n+1}^{p}$. The difference between this and the known viscoplastic strain tensor at $t_{n}$ given by

$$
\Delta \varepsilon_{n+1}^{p}=\varepsilon_{n+1}^{p}-\varepsilon_{n}^{p}
$$

must simultaneously be calculated from the constitutive equations given in Section 2 with all strain increments approximated by finite differences as in Eqn. (22).

The equations are nonlinear in $\Delta \varepsilon_{n+1}^{p}$ and are solved iteratively. In the ( $\left.i+1\right)$-th step in the iteration scheme between two succeeding time steps, we first calculate the displacement field $\left(U_{j_{n+1}}\right)_{i+1}$. The known $i$ th value

$$
\left(\varepsilon_{n+1}^{p}\right)_{i}=\varepsilon_{n}^{p}+\left(\Delta \varepsilon_{n+1}^{p}\right)_{i}
$$

of the viscoplastic strain field is here inserted in Eqn. (20). When $i=0$, the increment in viscoplastic strain at $t_{n+1}$ is given by

$$
\left(\Delta \varepsilon_{n+1}^{p}\right)_{0}=\frac{\Delta t_{n+1}}{\Delta t_{n}}\left(\Delta \varepsilon_{n}^{p}\right)_{\max }
$$


where the subscript 'max' indicates that convergence is obtained in the iterations at $t_{n}$. This is equivalent to assuming constant viscoplastic strain rate from one time step to the next.

Keeping $\left(U_{j_{n+1}}\right)_{i+1}$, and thereby also the total strain $\left(\varepsilon_{n+1}\right)_{i+1}$, constant, the next step is to update the viscoplastic strain increment $\Delta \varepsilon_{n+1}^{p}$ and the elastic strain $\varepsilon_{n+1}^{e}$. From Eqn. (7), we obtain

$$
\left(\varepsilon_{n+1}\right)_{i+1}=\left(\varepsilon_{n+1}^{e}\right)_{i+1}+\varepsilon_{n}^{p}+\left(\Delta \varepsilon_{n+1}^{p}\right)_{i+1}+\varepsilon_{n+1}^{T}+\varepsilon^{i}
$$

where all terms but $\left(\varepsilon_{n+1}^{e}\right)_{i+1}$ and $\left(\Delta \varepsilon_{n+1}^{p}\right)_{i+1}$ are known. Due to the incompressibility of the plastic deformation, only the deviatoric part of the elastic strain has to be considered, and the above equation can be reduced to

$$
\left(\varepsilon_{n+1}^{e^{\prime}}\right)_{i+1}+\left(\Delta \varepsilon_{n+1}^{p}\right)_{i+1}=\mathrm{C}
$$

where $\mathbf{C}$ is known. The deviatoric component of the elastic strain is denoted by the Superscript ". . According to Prandtl Reuss equations (Eqn. (11)), $\left(\Delta \varepsilon_{n+1}^{p}\right)_{i+1}$ has the same direction as the associated deviatoric stress vector. From Hooke's law (Eqns. (9) and (10)), we then see that $\left(\varepsilon_{n+1}^{e^{\prime}}\right)_{i+1}$ also has this direction. Hence,

$$
\left(\bar{\varepsilon}_{n+1}^{e^{\prime}}\right)_{i+1}+\left(\Delta \bar{\varepsilon}_{n+1}^{p}\right)_{i+1}=\bar{C}
$$

where the bar denotes effective values and where the first term on the left-hand side can be related to the effective flow stress by Hooke's law and the definitions of effective quantities. When the effective flow stress is interchanged with $f$ (defined by Eqn. (15), we finally obtain

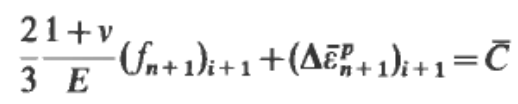

$\left(f_{n+1}\right)_{i+1}$ shall now be related to known quantities and $\left(\Delta \bar{\varepsilon}_{n+1}^{p}\right)_{i+1}$. Here, we approximate $\left(f_{n+1}\right)_{i+1}$ by the linear terms of a Taylor series around the value of the $i$ th iteration between time steps $t_{n}$ and $t_{n+1}$. Hence,

$$
\begin{aligned}
\left(f_{n+1}\right)_{i+1} \approx & \left(f_{n+1}\right)_{i} \\
& +\left(\frac{\partial f}{\partial \alpha}\right)_{i}\left[\left(\alpha_{n+1}\right)_{i+1}-\left(\alpha_{n+1}\right)_{i}\right] \\
& +\left(\frac{\partial f}{\partial \overline{\bar{\varepsilon}}^{p}}\right)_{i}\left[\left(\overline{\bar{\varepsilon}}_{n+1}^{p}\right)_{i+1}-\left(\overline{\bar{\varepsilon}}_{n+1}^{p}\right)_{i}\right]
\end{aligned}
$$

where we have used backward differences for the effective viscoplastic strain rate

$$
\left(\bar{\varepsilon}_{n+1}^{p}\right)_{i}=\frac{\left(\Delta \bar{\varepsilon}_{n+1}^{p}\right)_{i}}{\Delta t_{n+1}}
$$

and

$$
\left(f_{n+1}\right)_{i}=f\left[\alpha=\left(\alpha_{n+1}\right)_{i}, \quad \bar{\varepsilon}^{p}=\left(\bar{\varepsilon}_{n+1}^{p}\right)_{i}, \quad T=T_{n+1}\right]
$$

Mathematical expressions for the derivatives in Eqn. (29) can be found analytically since $f$ is known, and their subscript $i$ means that the $i$ th values of $\alpha_{n+1}$ and $\tilde{E}_{n+1}^{p}$ should be used in Eqn. (31). When $T \leqslant T_{0}$,

$$
\Delta \alpha_{n+1}=\alpha_{n+1}-\alpha_{n}=\Delta \bar{\varepsilon}_{n+1}^{p}
$$


in accordance with Eqn. (14), and it is easily seen that

$$
\left(\alpha_{n+1}\right)_{i+1}-\left(\alpha_{n+1}\right)_{i}=\left(\Delta \bar{\varepsilon}_{n+1}^{p}\right)^{i+1}-\left(\Delta \bar{\varepsilon}_{n+1}^{p}\right)_{i}
$$

It should, however, be noted that when $T>T_{0}, \Delta \alpha_{n+1}$ is equal to zero, leading to a zero second term on the right-hand side of Eqn. (29). The total viscoplastic strain is then generated by creep.

When the above expression for $\left(f_{n+1}\right)_{i+1}$ is inserted in Eqn. (28), we obtain an equation which is linear in the unknown $\left(\Delta \bar{\varepsilon}_{n+1}^{p}\right)_{i+1}$. In some cases when this equation is solved, it turns out that $\left(\Delta \bar{\varepsilon}_{n+1}^{p}\right)_{i+1}$ is negative due to the applied effective viscoplastic strain rate being too large. In these cases, the calculated effective strain increment is reset to the value calculated in the $i$ th iteration divided by some suitable factor. To obtain a rapid numerical convergence, we have found it convenient to choose this factor to be 5 .

To obtain the vectoral value of the associated viscoplastic strain increment, which is the final step in the scheme, we apply the condition that the two terms $\left(\varepsilon_{n+1}^{e^{\prime}}\right)_{i+1}$ and $\left(\Delta \varepsilon_{n+1}^{p}\right)_{i+1}$ on the left-hand side of Eqn. (26) have the same direction. Hence,

$$
\left(\Delta \varepsilon_{n+1}^{p}\right)_{i+1}=\frac{\mathbf{C}}{\bar{C}}\left(\Delta \bar{\varepsilon}_{n+1}^{p}\right)_{i+1}
$$

As a stopping criterion for the iterations, we apply that the difference between two successive values of the left-hand side of the above equation is smaller than a prescribed limit everywhere in the billet.

It should be noticed that any function $f$ containing any internal variable (in our case called $\alpha$ ) can, in principle, be applied in the above algorithm provided that its derivatives with respect to $\alpha$ and $\bar{\varepsilon}^{p}$ are known. Furthermore, from a mathematical and numerical point of view, it is very easy to introduce more than one internal variable into our theory, leading correspondingly to more first-order terms on the right-hand side of Eqn. (29).

With our specific choice of $f$, i.e., Eqns. (15) to (18), the derivative of $f$ with respect to $\bar{\varepsilon}^{p}$ approaches infinity when $\bar{E}^{p}$ approaches zero, reflecting the inherent stiff characteristics mentioned above. In our case, this problem is solved by giving each component of $\bar{\varepsilon}^{p}$ the value $10^{-6} \mathrm{~s}$ when an integration point is incorporated into the solution domain.

In the limit of time-independent plasticity, a yield criterion must be incorporated in the mathematical description, and a minor modification of the numerical scheme is required. First, the final term on the right-hand side of Eqn. (29) is omitted. Second, the yield criterion is incorporated by checking whether the effective plastic strain increment calculated by solving Eqn. (29) is positive or negative. In the former case, we have yield, and we can continute the calculations immediately. In the latter case, the calculated effective plastic strain increment must be reset to zero.

We have shown numerically that our scheme can handle a material description where the plastic strain has a very small time dependency. For example, by letting $m$ in Eqn. (15) equal 0.01, which approximates to a time-independent case, the scheme may still be applied without any modifications. Within the numerical accuracy, we then obtain results equivalent to $m$ equal to zero with the yield criterion implemented. Since $m>0.01$ for the AlMgSi alloy AA6063, it has not been necessary to modify the numerical code in the present study.

Two test cases have been carried out in order to verify the numerical scheme discussed above. In these cases, we have used 'simplified' material descriptions to which other numerical solution algorithms apply. Otherwise, the two cases are similar to the 
stress problem which is defined in Section 2, and approximately 1 minute of the DC casting process is simulated. Compared to the material data for an aluminium alloy, the material properties in the 'simplified' material descriptions are erroneous. However, these errors do not have any consequences for the adequacy of the tests, since the same material description is used both in the test case and in the algorithm that is to be tested.

Implementing the yield criterion in the ALSPEN algorithm, a purely timeindependent case has first been modeled by setting $m \equiv 0$ in Eqn. (15). The calculated results are equivalent to those we have obtained by using a numerical solution algorithm very similar to that described in Chapter 7 in Owen and Hinton (1980).

In the second case, a material model accounting only for creep in the generated viscoplastic strain was applied ( $n \equiv 0$ in Eqn. (15)). To verify the results obtained by the ALSPEN algorithm, we also solved the equations for 'pure creep' using explicit (and, in our case, very time-consuming!) time integration, as described in Zienkiewitcz and Cormeau (1974). Also, in this case, a complete agreement was obtained.

\section{Results}

\section{4(A) Casting conditions and thermal modeling}

In the following case study of the DC casting of an AlMgSi alloy 6063, the billet radius equals $97.5 \mathrm{~mm}$, and the casting speed is equal to $1.67 \mathrm{~mm} / \mathrm{s}$. Between the billet and the mould and between the billet and the bottom block, air gaps may form as a result of thermal contraction, and the results from the thermal modeling are locally strongly affected by the extent of these air gaps. In the boundary conditions of the model ALSIM-2, the development of an air gap between the billet and the bottom block has been taken into account by implementing time-dependent coefficients for surface heat transfer (Jensen and Schneider 1990). We have furthermore accounted for an air gap between the billet and the mould when the temperature inside the billet in a normal distance of $5 \mathrm{~mm}$ from $\Gamma_{4}$ is below $908 \mathrm{~K}$. By this criterion, the thickness of the solid metal close to $\Gamma_{4}$ is assumed to be large enough to withstand the pressure from the liquid melt, and the air gap is assumed to extend from the bottom of the mould to the uppermost point along $\Gamma_{4}$ in which the criterion defined above is fulfilled. A more detailed discussion of this boundary condition can be found in Jensen (1980). In the present versions of the models, there exists no mathematical backcoupling between ALSPEN and ALSIM-2 to account for changes in the geometry of the air gaps as computed by ALSPEN. For a detailed treatment of this problem, we refer to Kelly, Michalek, O'Connor, Thomas and Dantzig (1988). Except for the influence that change in the air gaps may have on the heat transfer, the calculation of the thermal history is independent of the stress calculation (Weckman and Niessen 1984).

The numerical results show a discrepancy between the extent of the air gaps calculated by ALSPEN and those accounted for in ALSIM-2. As an example, we may notice that for stationary casting conditions, ALSPEN predicts an air gap along the entire boundary $\Gamma_{4}$, i.e., between the solid part of the billet and the mould. In the ALSIM-2 calculation, however, the coefficients for surface heat transfer along $\Gamma_{4}$ reflect an air gap only up to a point approximately $5 \mathrm{~mm}$ below the solidification front $\Gamma_{5}$. The discrepancies in air gaps along $\Gamma_{4}$ reflect the mathematical coupling not accounted for in the present versions of ALSPEN and ALSIM-2. Most likely, however, there is another and possibly more important source for the inaccurate result in the example discussed above - namely, the constitutive description. Along the upper part of $\Gamma_{4}$. there are very high temperatures for which we believe that the material model needs further development. 
For further details about the thermal modeling prior to the present stress modeling, we refer to Jensen and Schneider (1990), and we refer to Vorren and Brusethaug (1987) for a more detailed discussion of the different boundary conditions available in ALSIM-2. Additional information necessary for the stress modeling, i.e., the boundary conditions, the mechanical material description, and the coherency temperature, $T_{c}$, have been given in the preceding sections and in references cited there.

\section{4(B) Numerical accuracy}

In the finite-element solution, we have applied 20 elements in the radial direction, and the element mesh is shown in Fig. 1. The height of the elements and the time step are chosen to be $3 \mathrm{~mm}$ and $0.4 \mathrm{~s}$, respectively, and the limit defining the convergence criterion for the iterations on viscoplastic strain increment discussed in Section 3(C) is set to $2 \times 10^{-6}$.

The discretization gives a numerical accuracy of $2 \%$ or better in all quoted values for the stresses, displacements, and strains, except for quantities calculated in elements at the boundary $\Gamma_{5}$. These quantities, however, are very close to zero, and the errors have no influence on the accuracy in any other elements. To model 6 min and $20 \mathrm{~s}$ casting time, ALSPEN needs approximately 13 hours CPU time on a VAXSTATION $\dagger$ 3100 .

\section{4(C) Stationary casting conditions}

After a casting time of 6 to $7 \mathrm{~min}$, approximately stationary casting conditions have been attained, and the stress modeling can be terminated. At this time, the calculated quantities in any given position from the mould are (approximately) unchanged as time proceeds, and the lower part of the billet has reached a temperature below $50^{\circ} \mathrm{C}$, i.e., the temperature in the surrounding cooling water.

Figurcs $2(a)(d)$ show contour maps of the four components of the thermal stresses under stationary cast conditions. $\neq$ (The corresponding contour map of the temperature distribution is given in Fig. 4(a).) We note that the residual radial stresses, displayed in the part of Fig. $2(a)$ where the temperature is below $50^{\circ} \mathrm{C}$, are positive and increase monotonically with the depth into the billet. The circumferential and the axial components of the residual stresses, displayed in Figs $2(b)$ and $(c)$, change sign for $r$ approximately equal to 40 and $60 \mathrm{~mm}$, respectively. These results are in qualitative agreement with measurements (Roth, Welsch and Röhrig 1942). Finally, we see that the water impingement point at $\Gamma_{3}, 4 \mathrm{~mm}$ below the mould, has a significant effect on the stress distribution, especially the circumferential component.

Contour maps of the viscoplastic part of the strain distribution at stationary casting conditions are shown in Figs $3(a)-(d)$. We see that the radial component is relatively homogeneous and that the circumferential component changes from tension to compression with $r$. It should be noticed that the relatively large compressive value of

†VAXSTATION is a trademark of Digital Equipment Corporation, Maynard, MA.

$\ddagger$ The apparent tensile radial stress at the solidification front $\Gamma_{5}$ near the centreline is just an artifact of the resolution of the applied numerical method. Compared to the considerable tensile radial stress which is developed near the centreline immediately after the metal has solidified, the small compressive hydrostatic pressure at $\Gamma_{5}$ from the above liquid melt is almost negligible. Also, the apparent nonzero shear stresses along the lower part of the centreline $\Gamma_{1}$ are an artifact of the resolution of the applied numerical method. In accordance with the symmetry condition, $\sigma_{p z}$ is zero at $\Gamma_{1}$. 
$\widehat{E}$
$\frac{n}{x}$
$\frac{1}{N}$
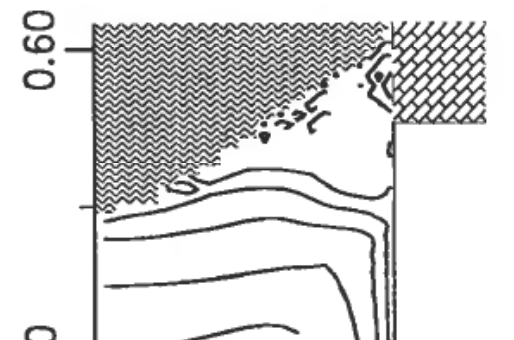

융

임

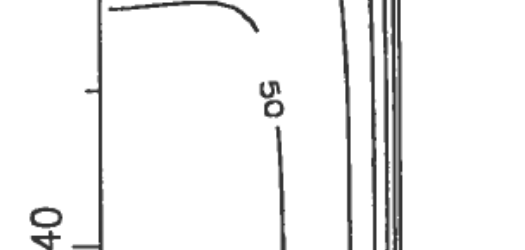

\&

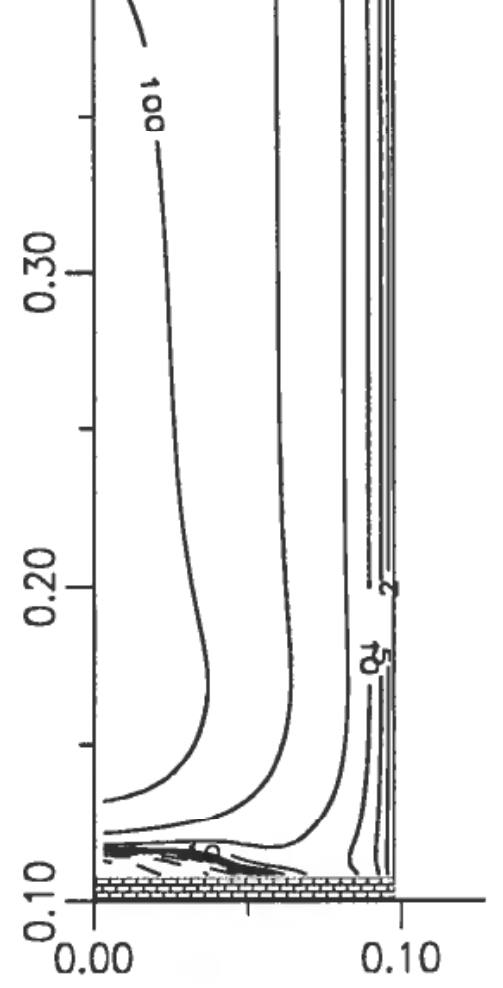

R-Axis (m)

(a)

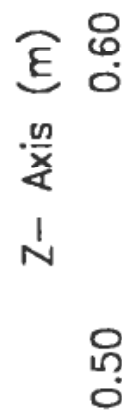
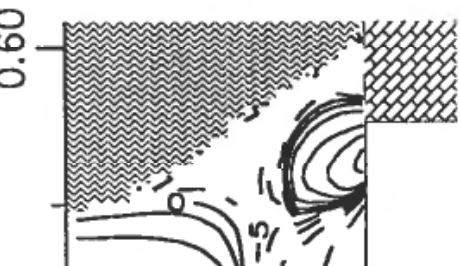
in

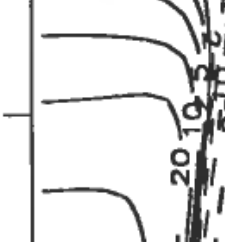

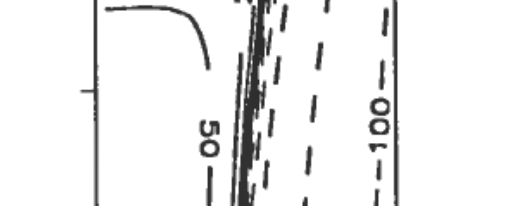

웅

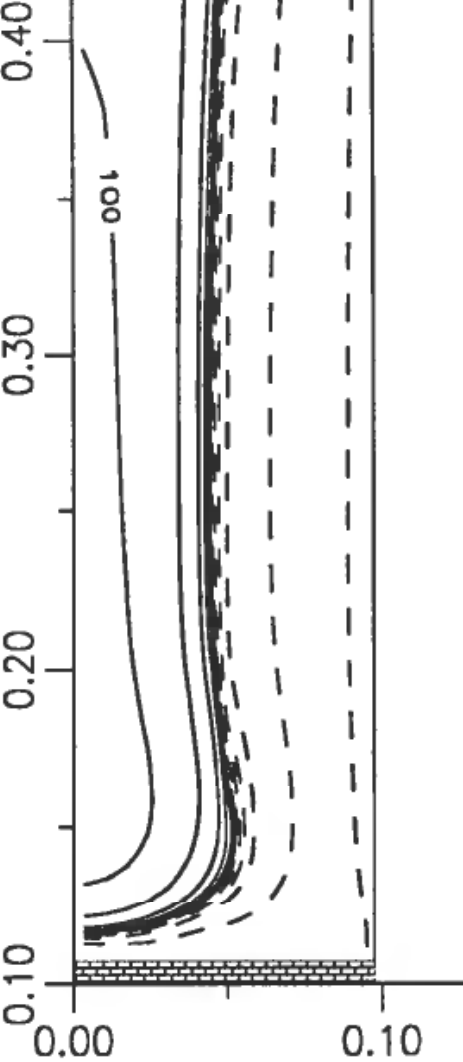

R- Axis (m)

(b) 


$$
\frac{\mathscr{E}}{\mathrm{E}}
$$

$E$
$\frac{n}{x}$
N

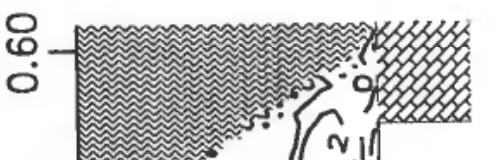

E्
$\frac{n}{x}$
N
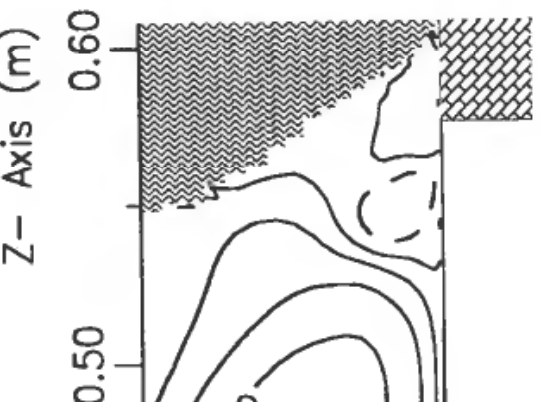

눙
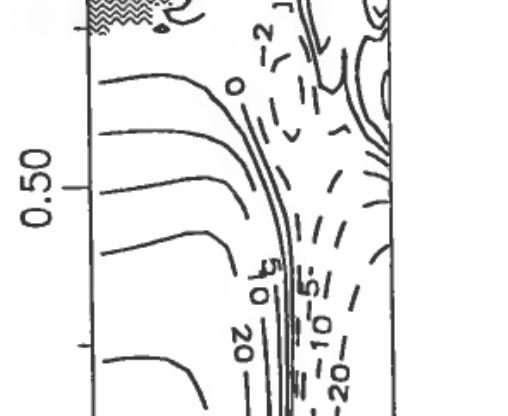

웅 n:

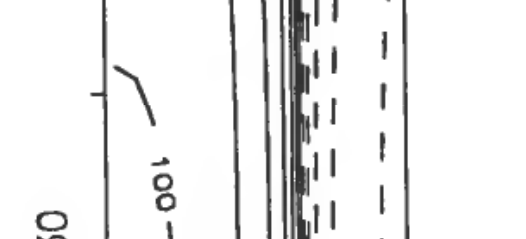

m

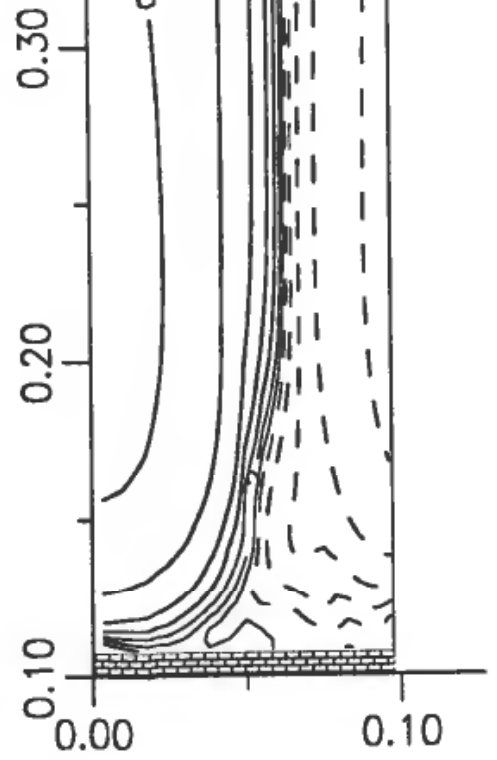

$\mathrm{R}-\operatorname{Axis}(\mathrm{m})$

(c)

Figure 2. Thermal stresses in MPa at stationary casting conditions. (a) Radial component, (b) circumferential component, $(c)$ axial component, and $(d)$ shear component. Solid and dashed lines correspond to tension and compression respectively. 


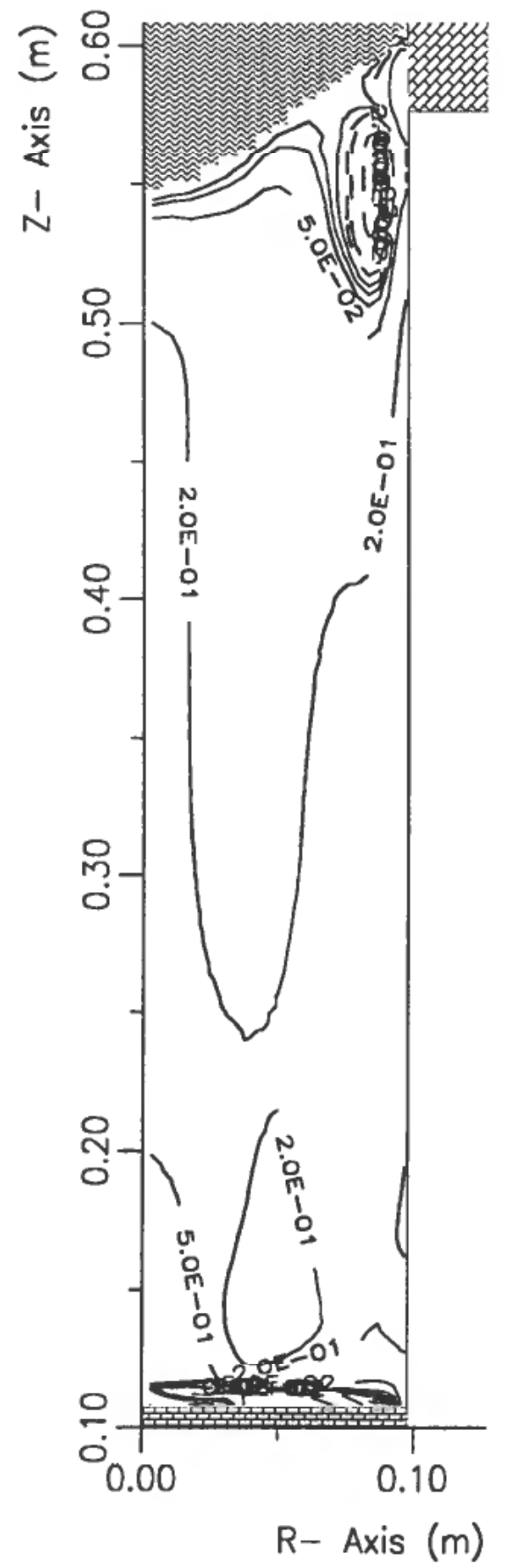

(a)

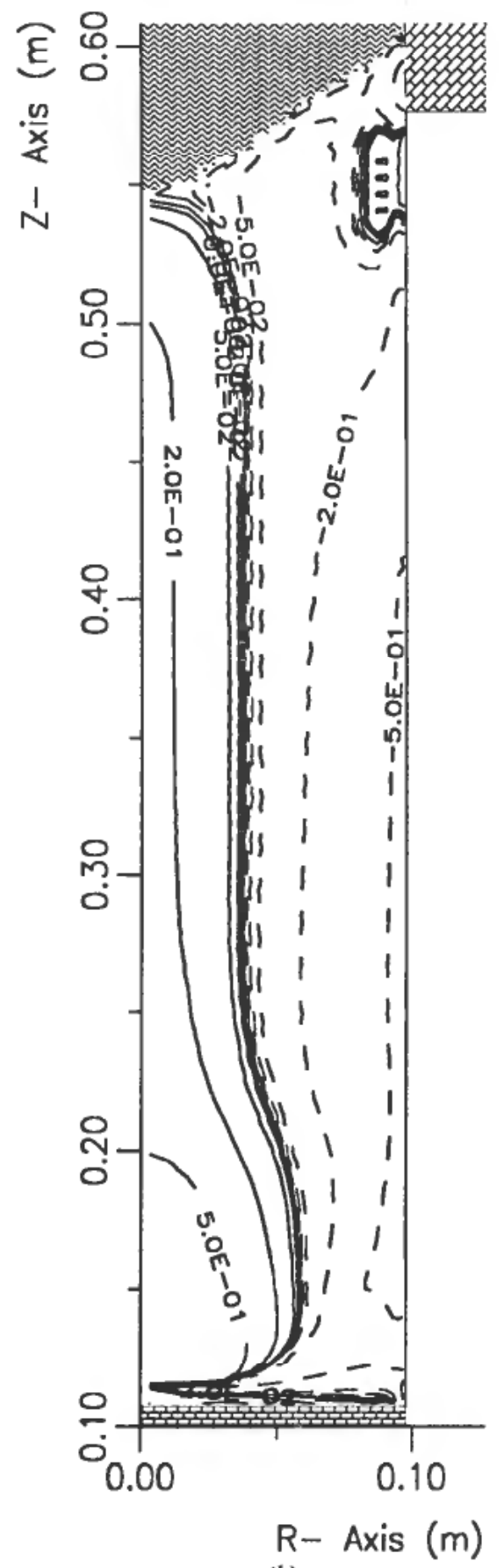

(b) 


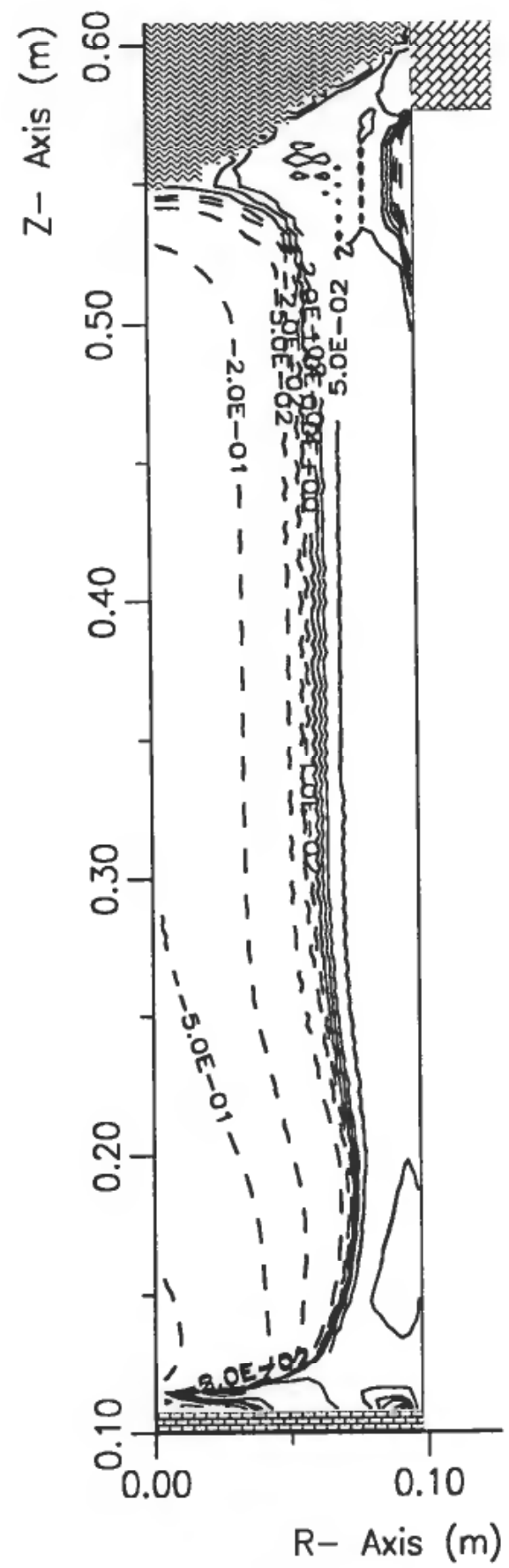

(c)

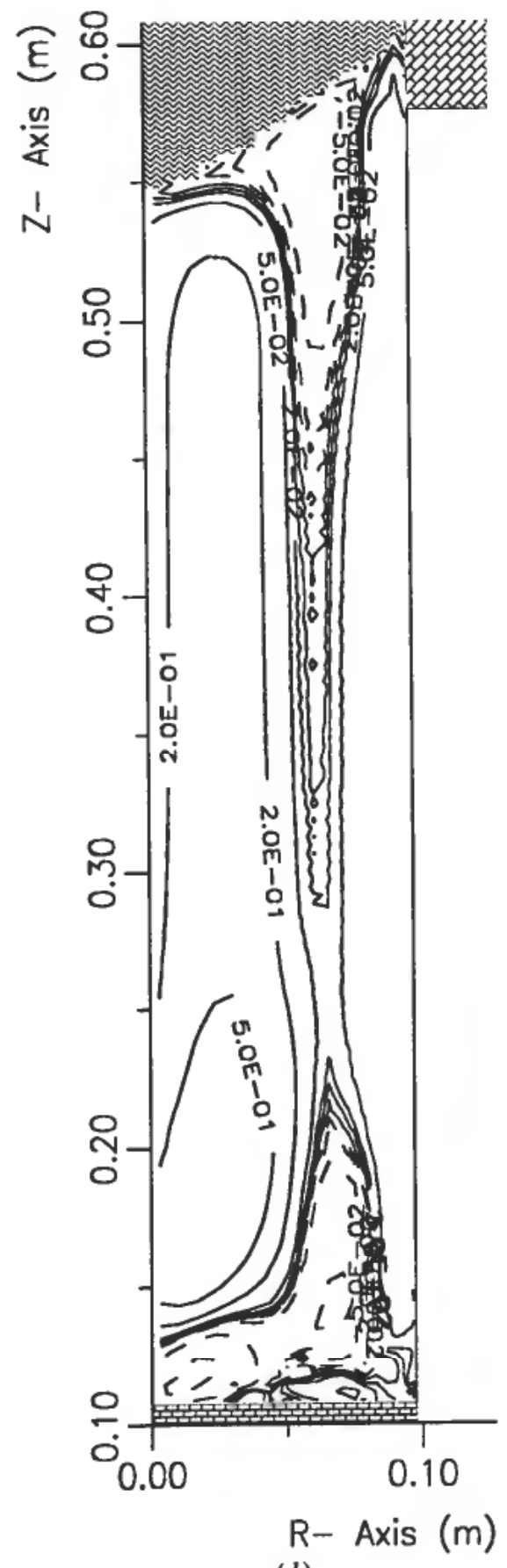

(d)

Figure 3. Viscoplastic strain in $\%$ at stationary casting conditions. (a) Radial component, (b) circumferential component, $(c)$ axial component, and $(d)$ shear component. Solid and dashed lines correspond to tensional and compressive strain values respectively. 
the circumferential component near the outside surface of the billet accounts for an important part of the total radial contraction of the billet during casting.

The axial component of the viscoplastic strain in the lower part of the billet changes from compression to tension with $r$. This result may seem unexpected when it is compared to the axial stress component of the stress tensor, which changes from tension to compression (Fig. $2(\mathrm{c})$ ). Concerning the region near the centreline, however, it is a consequence of a large compressive axial viscoplastic strain rate near the solidification front $\Gamma_{5}$. As will be further discussed in Section 4(D), almost all of the viscoplastic strain is generated by creep at high temperatures near the centreline. In other words, the compressive axial component of the (accumulated) viscoplastic strain closely to $\Gamma_{1}$ in the 'cold' part of the billet reflects a relatively large axial component of the viscoplastic strain rate, when this part of the billet was 'warm'. Furthermore, according to Prandtl-Reuss relations (Eqn. (11)), the sign of the axial viscoplastic strain rate equals the sign of the deviatoric axial stress component, which is given by

$$
\sigma_{z}^{\prime}=\sigma_{z}-1 / 3\left(\sigma_{r}+\sigma_{\theta}+\sigma_{r}\right)
$$

and in regions near the corner defined by $\Gamma_{5}$ and $\Gamma_{1}, \sigma_{z}^{\prime}$ is negative since $\sigma_{r} \approx \sigma_{\theta}>\sigma_{z}>0$ (Fig. 2(c)).

Also, in the region near the outside surface of the billet, the axial components of the viscoplastic strain and stress have different signs, and this result may be explained by studying the axial component of the viscoplastic strain rate.

\section{4(D) Development of plastic yield}

The development of plastic yield is examined in Figs $4(a)-(e)$. Here, we have displayed contour maps for stationary casting conditions of the temperature $T$, the effective viscoplastic strain rate $\bar{\varepsilon}^{p}$, the hardening parameter $\alpha$, the flow stress $\bar{\sigma}$, and the accumulated effective viscoplastic strain $\int d \bar{\varepsilon}^{p}$. We note first that there are both 'hightemperature regions' $\left(T>T_{0}=700 \mathrm{~K}=427^{\circ} \mathrm{C}\right)$ and 'low-temperature regions' $\left(T<T_{0}\right)$ in the billet where $\bar{\varepsilon}^{p}$ is significant (i.e., larger than $10^{-4}$ ). Hence, a description of the plastic material properties is necessary at all temperatures. If, on the other hand, no yield were present below, for example, $T_{0}$ (which may be regarded as the onset temperature for strain hardening), the total viscoplastic strain would have been generated by creep only. In that case, a general viscoplastic description valid in the entire temperature range would not have been necessary.

The regions in Fig. 4(c) with a nonzero $\alpha$ value reflect the strain hardening generated in the billet. One result of the strain hardening is seen in Fig. 4(d), where the residual flow stress in some regions is greater than $70 \mathrm{MPa}$, which is the (effective) initial yield stress for aluminium at room temperature. We also note that in the centre of the billet almost all of the viscoplastic strain is due to creep at high temperatures since $\alpha$ here is very low. Finally, from Fig. (4(e), it is noted that the maximum effective viscoplastic strain generated in the billet is approximately $2 \%$.

In our material description, the temperature $T_{0}=700 \mathrm{~K}$ has been introduced as an onset temperature for strain hardening, and the effect of strain hardening may therefore be easily studied by changing the value of $T_{0}$. In Figs $(5(a)$ through $(c)$, we have compared the residual stress variations with radii in central regions of the billet at stationary casting conditions with value obtained by choosing $\mathrm{T}_{0}$ equal to $400 \mathrm{~K}$. It is seen that by neglecting the strain hardening in the temperature range between $700 \mathrm{~K}$ and $400 \mathrm{~K}$, the maximum value of the residual stresses decreases by 7 to $8 \%$. 


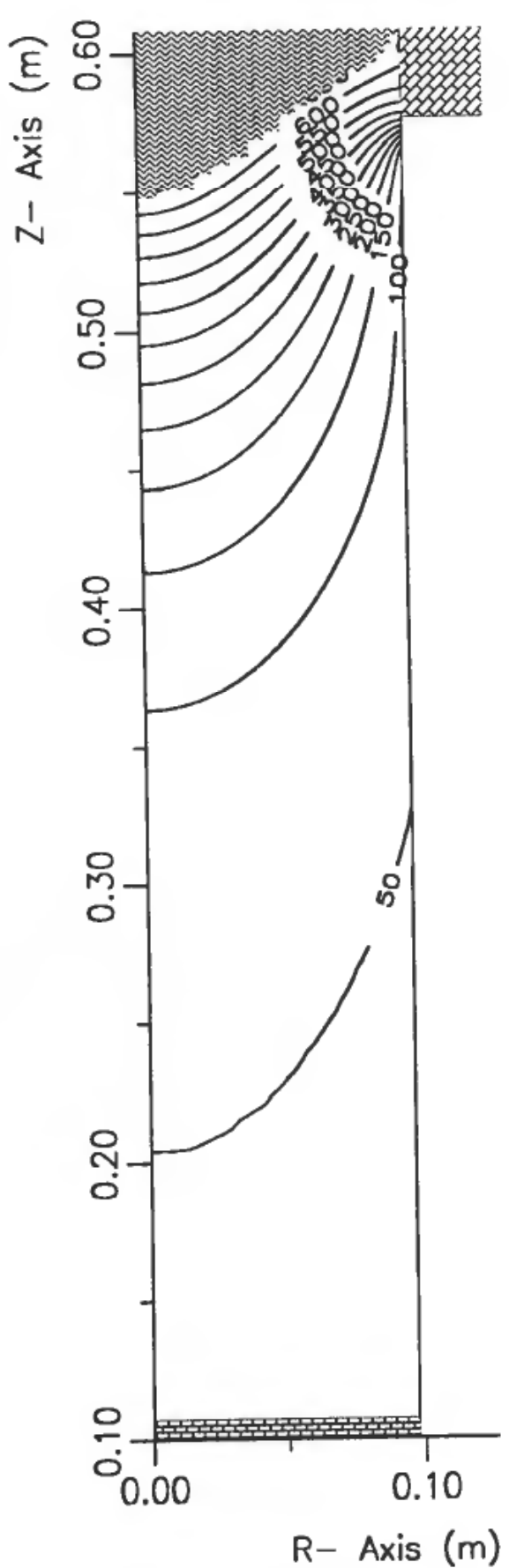

(a)

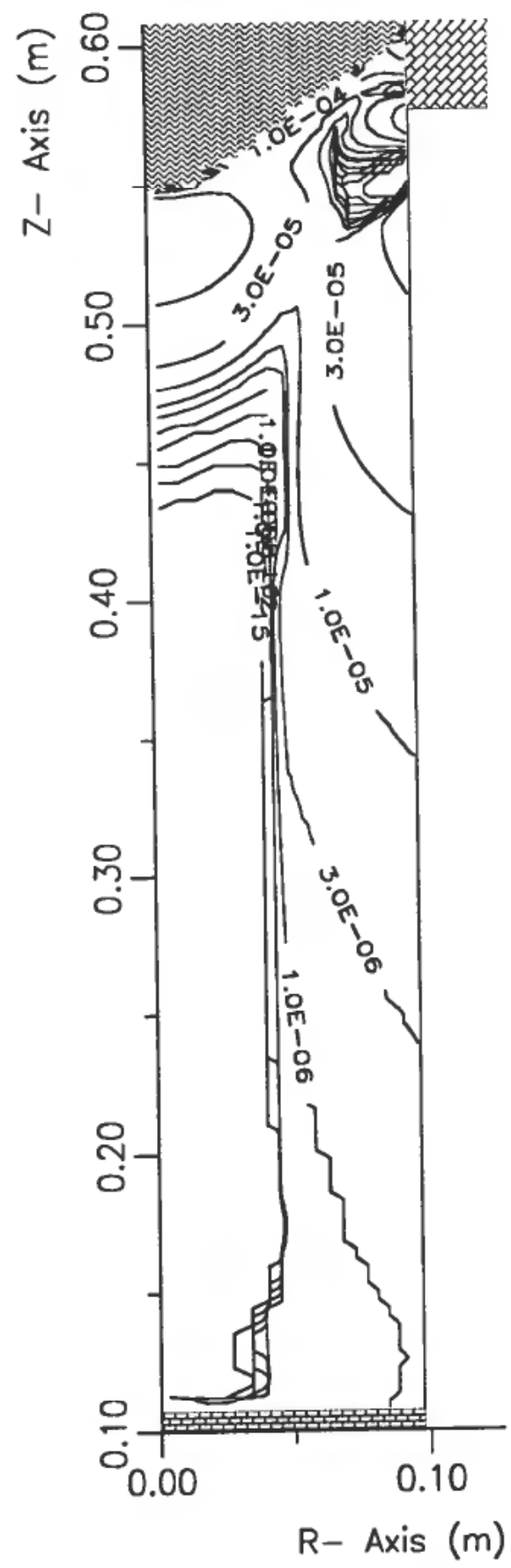

(b) 


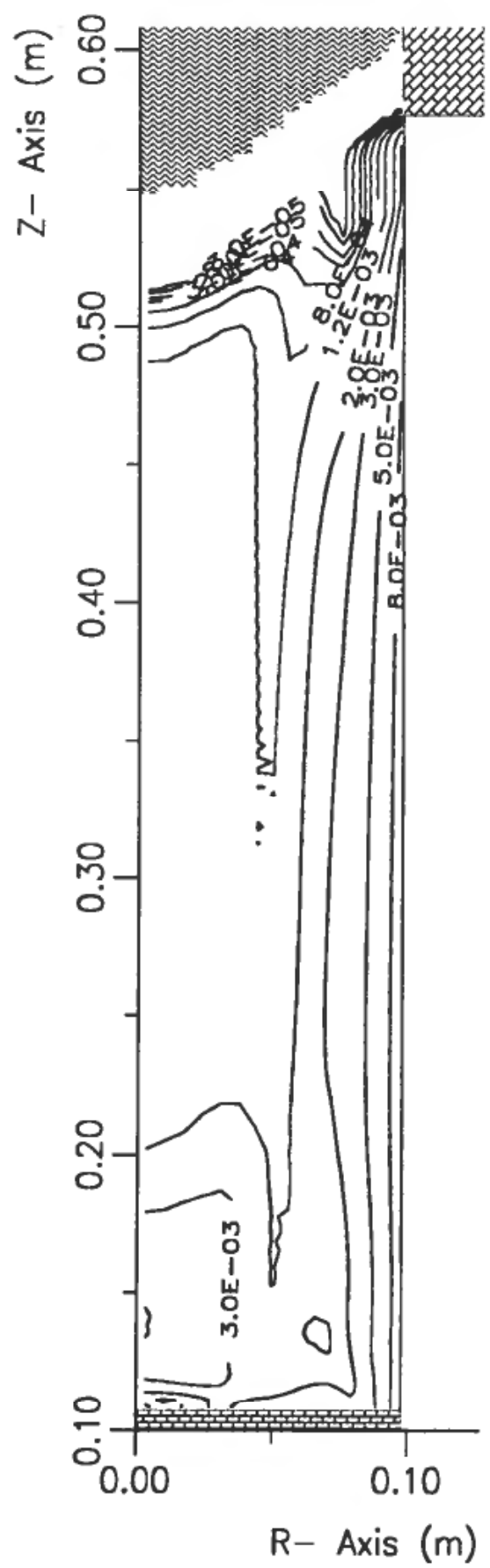

(c)

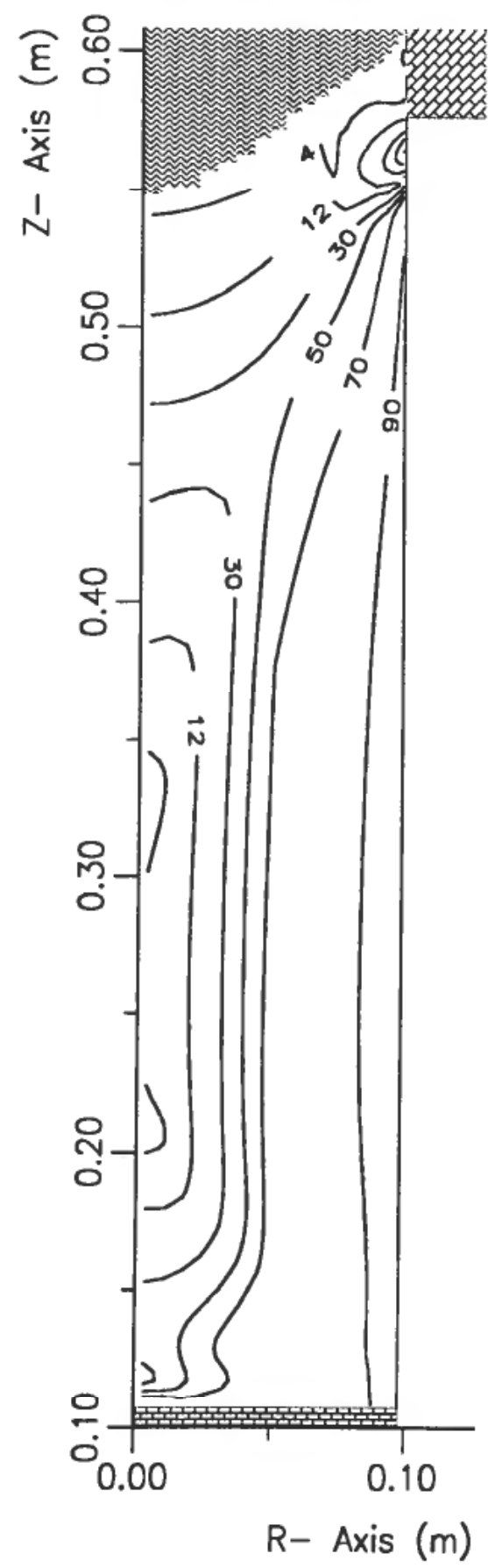

(d) 


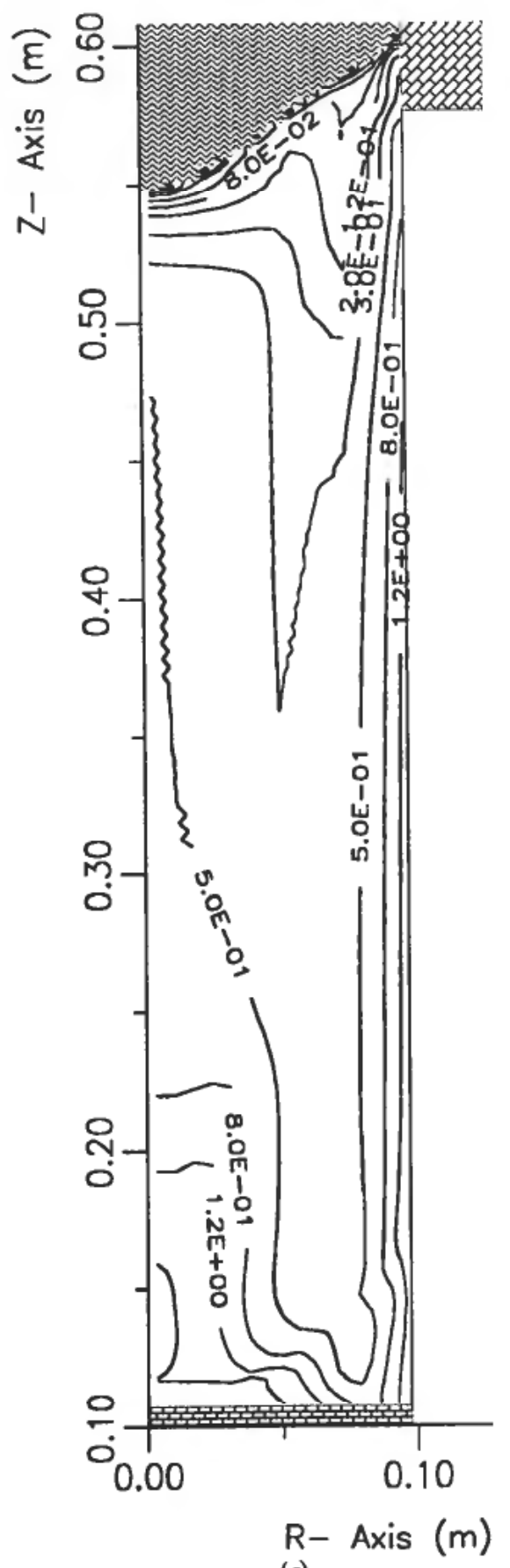

(e)

Figure 4. Development of plastic yield, isoplots at stationary casting conditions of: (a) temperature in ${ }^{\circ} \mathrm{C},(b)$ effective viscoplastic strain rate, $(c)$ hardening parameter, $(d)$ effective stress in $\mathrm{MPa}$, and $(e)$ integrated effective viscoplastic strain in \%. In the black regions in (c), $T>T_{0} \Rightarrow \alpha \equiv 0$.) 


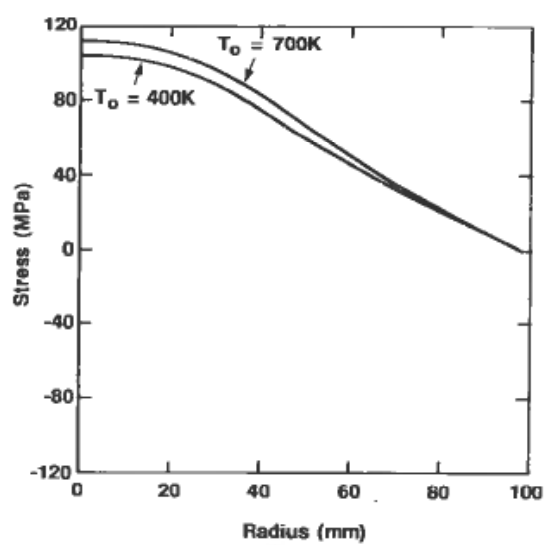

(a)

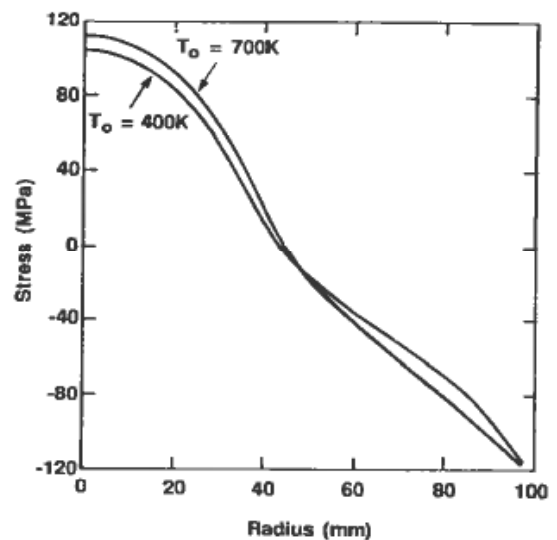

(b)

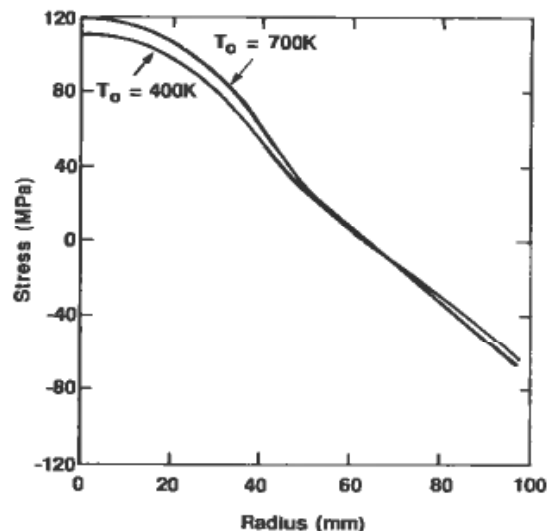

(c)

Figure 5. The effect of work hardening on residual stresses. (a) Radial component, (b) circumferential component, and (c) axial component versus radius for two values of $T_{0}$.

\section{4(E) Air gap formation, billet-bottom block}

The development of an air gap between the billet and the bottom block can be studied in Figs 6(a)-(c), where we have shown the displaced element mesh after 20 and 40 seconds casting time and at the end of casting. In accordance with observations (Jensen 1989) it is seen that an air gap is formed when the cooling water starts to impinge on the billet. Also, the curved bottom profile of the finished fillet shown in Fig. 6(c) is observed in castings (Jensen 1989).

In Jensen and Schneider (1990) it is discussed how this air gap has been accounted for in the thermal modeling on which the present stress modeling is based.

\section{Concluding remarks}

In the present paper, we have discussed the mathematical model ALSPEN by which we have calculated the thermally induced strains and stresses in a DC casting of an AlMgSi alloy 6063. Our numerical results are in agreement with observations of the casting process, and the calculated residual stresses are in qualititive agreement with measurements available in the literature. 


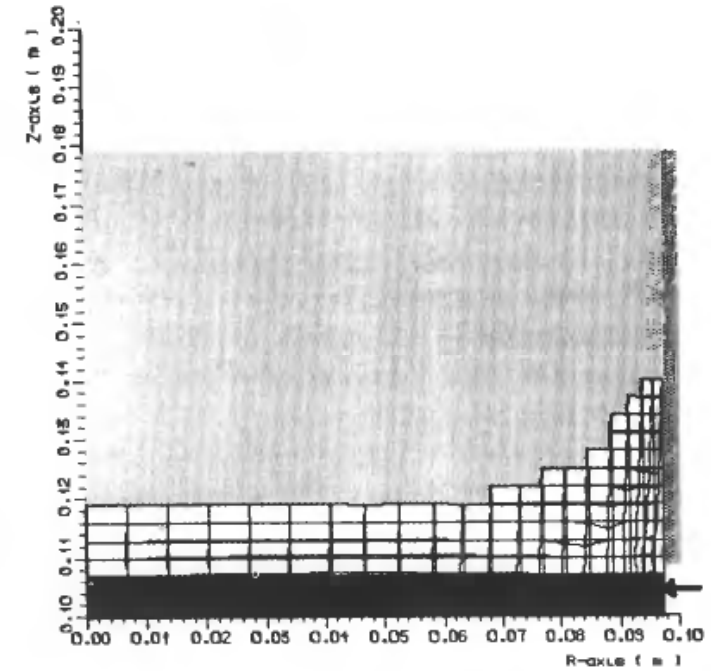

(b)

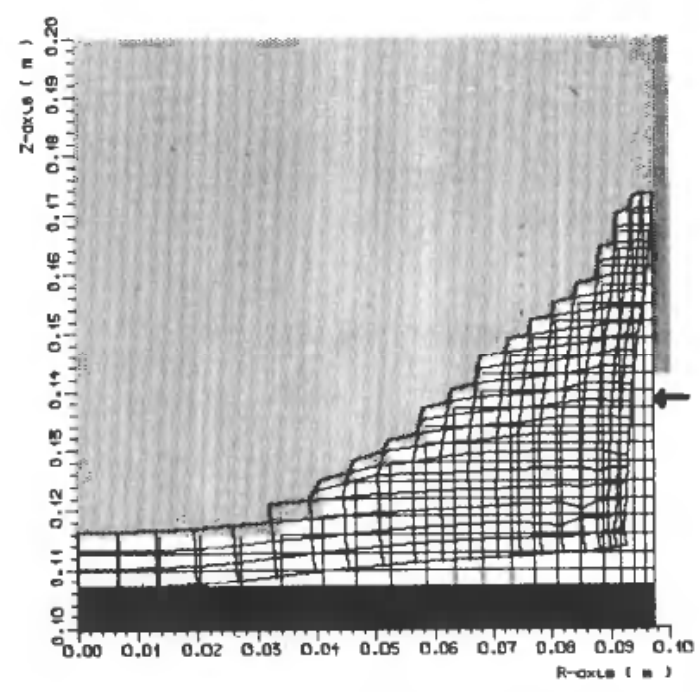

(c)

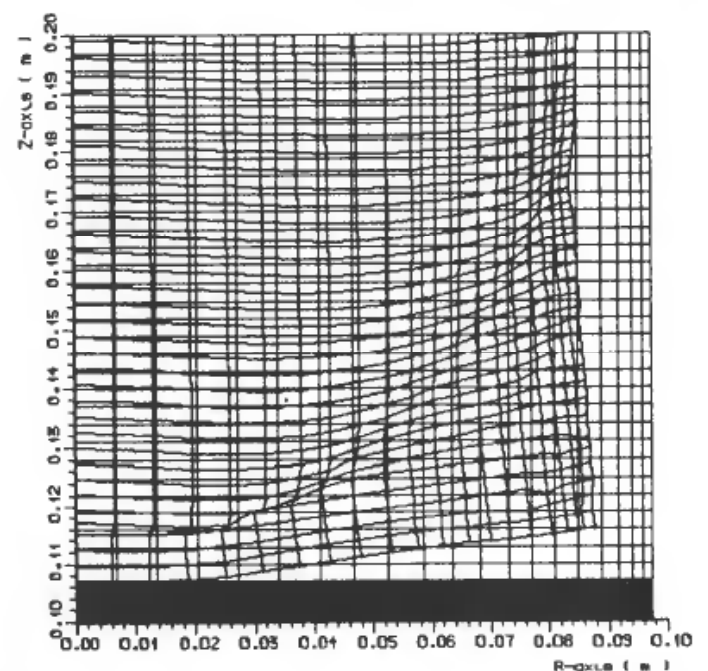

Figure 6. Development of an air gap between the billet and the bottom block. (a) Displaced element mesh after $20 \mathrm{~s}$ casting time, $(b)$ after $40 \mathrm{~s}$ casting time, and $(c)$ at the end of casting. The displacements are magnified with a factor of 5 for visualization. The arrow indicates the impingement point of the cooling water. 
At the present stage, it is of prime importance to establish a quantitative verification of the calculated results by comparing them with measurements. Another important topic for our research is to apply the numerical results as input to criteria for hot tearing and cracking.

\section{ACKNOWLEDGMENTS}

One important basis for the present paper is the collaboration with $\mathbf{M}$. L. Nedreberg, MS, and her supervisor, Professor B. Andersson, both at Senter for Industriforskning (SI). We also wish to acknowledge Siv. ing. E. K. Jensen at Elkem Aluminium for valuable suggestions, and Dr. J. E. Tibbals, SI, for stimulating discussions and for correcting the manuscript. The development of ALSPEN was funded by Hydro Aluminum, Elkem Aluminum and The Royal Norweigan Council for Scientific and Industrial Research (NTNF).

\section{REFERENCES}

Agyris, J. H., VAZ, L. E. and WiLliam, K. J., (1978). Comput. Methods Appl. Mech. Eng., 16, 23177.

Bammann, D. J. (1988). In Modeling and Control of Casting and Welding Processes IV, A. F. Giamei and G. J. Abbaschian, eds. (TMS, Warrendale, PA), pp. 329-38.

Brown, S. B., Kim, K. H. and ANAND, L. (1989). Int. J. Plasticity, 5, 95-130.

Claxton, R. J. (1973). In Continuous Casting, K. R. Olen, ed. (TMS-AIME, New York, NY), pp. $341-52$.

Crook, A. J. L. and Hinton, E. (1987). In Computational Plasticity: Models, Software and Applications, D. R. J. Owen and E. Hinton, eds (Pineridge Press Limited, Swansea), pp. $435-48$.

Fossheim, H. and Madsen, E. E. (1979). In Light Metals, W. S. Peterson, ed. (TMS-AIME, Warrendale, PA), pp. 695-720.

JENSEN, E. K. (1980). In Light Metals, K. J. McMinn, ed.(TMS-AIME, Warrendale, PA), pp. 631 42.

JENSEN, E. K. and SCHNeIDER, W. (1990). In Light Metals, Christian M. Bickert, ed. (TMS-AIME, Warrendale, PA), pp. 937-43.

JENSEN, E. K. (1989). Elkem Aluminum, Farsund, Norway, private communication.

Kelly, J.E., Michalek, K. P. O'ConNor, T. G., Thomas, B. G. and DANTZIG, J. A. (1988). Metall. Trans. A, 19A, 2589-2602.

Kristiansson, J. O. (1982), J. Therm. Stresses, 5, 315-30.

Kristiansson, J. O. and ZetTERLund, E. H. (1982). In Numerical Methods in Industrial Forming Processes, (Pineridge Press Limited, Swansea), pp. 413-423.

Liu, W. K. Belytschko, T., Ong, J. S. J. and Law, E. S. (1985), Eng. Comput., 2, 47-55.

LiU, W. K. ONG, J. S. J. and UraS, R. A. (1985) Comput. Methods Appl. Mech. Eng., 53, 13-46.

Lush, A. M., Weber, G. and ANand, L. (1989). Int. J. Plasticity, 5, 521-49.

MaDSEN, E. E., (1979). In Numerical Methods in Thermal Problems, R. W. Lewis and K. Morgan, eds. (Pineridge Press Limited, Swansea), pp. 81-89.

Madsen, E. E. and Fladmark, G. E. (1973). In Numerical Solution of Partial Differential Equations, G. E. Fladmark and J. G. Gram, eds. (D. Reidel Publishing Company, Dordrecht), pp. 223-40.

Mahin, K. W., MacEwen, S., Winter, W., Mason, W., Kanouff, M. and Fuchs, E. A. (1988). In Modeling and Control of Casting and Welding Processes IV (TMS, WARRENDALE, PA), pp. 339-50.

Mathew, J. and Brody, H. D. (1976), Nucl. Metall, 20, pp. 978-90. 1979, Proc. Int. Conf. on Solidification, A. Nicholson, ed. (TMS, Warrendale, PA), Book 192, pp. 244-49.

Milzer, A. K. ed. (1987), Unified Constitutive Equations (Elsevier Applied Science, London).

Mondolfo, L. F. (1976). Aluminum Alloys, Structure and Properties (Butterworth's, London), pp. 61 and $81-82$

MORICEAU, J. (1975). In Light Metals, R. Rentsch, ed. (TMS-AIME, WARRENDALE, PA), vol. 2, pp. 119-33. 
Nedreberg, M. L. (1990). Ph.D. Thesis, University of Oslo, Oslo, Norway.

OWEN, D. R. J. and Hinton, E. (1980). Finite Elements in Plasticity, 1st ed. (Pineridge Press Limited, Swansea).

Perzyna, P. (1966). Adv. Appl. Mech., 9, 243-377.

Pierce, D. Shih, C. F. and Needleman, A. (1984). Comput. Struct., 8, 875-87.

RAMmerstorfer, F. G., JAQUemar, CH., Fisher, D. F. and H. Wieseinger (1979). In Numerical Methods in Thermal Problems, R. W. Lewis and K. Morgan, eds. (Pineridge Press Limited, Swansea), pp. 712-22.

Richmond, O. (1981). In Modeling of Casting and Welding Processes, H. D. Brody and D. Apelian, eds. (TMS, Warrendale, PA), pp. 215-22.

Roth, A., WelsCh, M. and RöHrIG, H. (1942). Aluminum, June/July, 206-09.

Sample, V. M. and Lalli, L. A. (1987). Mater. Sci. Technol., 3, 28-35.

SMELSER, R. E., and Richmond, O. (1988). In Modeling and Control of Casting and Welding Processes IV, A. F. Giamei and G. J. Abbaschian, eds. (TMS, Warrendale, PA), pp. 313-28.

SNYDER, M. D. and BATHE, K. J. (1981). Nucl. Eng. Des., 64, 49-80.

TanaKa, T. G. and Miller, K. 1988, Int. J. Numer. Methods Eng., 26, 2457-85.

Thomas, B. G., SamaraseKaera, I. V., and Brimacombe, J. K., 1987, Metall. Trans. B, 18B, 13147.

VoRREN, O. and BRUSETHAUG, S. (1987). In 8th Internationale Leicht Metall Tagung, Leoben and Vienna, Austria (Aluminium Verlag, Düsseldorf, Federal Republic of Germany, pp. 27883.

Weckman, D. C. and Niessen, P. (1984), Can. Metall. Q., 23, 209-16.

Williams, J. R., LeWIS, R. W. and MorGaN, K. (1979). Int. J. Numerical Method Eng., 14, 1-9.

Zienkiewitcz, O. C. and Cormeau, I. C. (1974). Int. J. Numer. Methods Eng., 8, 821-45. 